\title{
Mudanças ocorridas na composiçáo florística em decorrência da exploração florestal em uma área de floresta de Terra firme na regiáo de Paragominas, PA
}

Luciana Maria de Barros FRANCEZ1 , João Olegário Pereira de CARVALHO², Fernando Cristóvam da Silva JARDIM $^{3}$

\section{RESUMO}

O objetivo da presente pesquisa foi avaliar as mudanças ocorridas na composição florística, considerando duas intensidades de colheita de madeira, em 108 ha de floresta, na Fazenda Rio Capim, pertencente à Cikel Brasil Verde Madeiras Ltda., município de Paragominas, Estado do Pará. Os dados foram coletados, em duas ocasiōes (2003, antes da exploração e em 2004, após a exploração) em 36 parcelas permanentes quadradas de 0,25 ha, estabelecidas aleatoriamente na área, sendo doze para estudar a floresta nãoexplorada, doze para a explorada com colheita apenas do fuste comercial das árvores e doze para a explorada com colheita do fuste e dos resíduos lenhosos. Todos os indivíduos com DAP $\geq 10 \mathrm{~cm}$ foram registrados. Antes da exploração, ocorreram 4469 árvores nas 36 parcelas amostradas (nove hectares), distribuídos em 46 famílias, 138 gêneros e 228 espécies. Após a exploração, foram registrados 4330 indivíduos, porém duas espécies desapareceram (Licaria sp. e Nectandra sp.). A composição florística, nas duas intensidades de colheita, sofreu alterações significantes devido à exploração de impacto reduzido a que foi submetida. Entretanto, não houve alteraçóes significantes entre as duas áreas, demonstrando que, em termos ecológicos, a retirada dos resíduos lenhosos após a colheita dos fustes não implicou em danos significativos à floresta remanescente. Após a exploração, a composição florística, mesmo com pequenas alterações, não mostrou significância entre as três comunidades, sugerindo que com a intensidade de exploração aplicada, mais a retirada adicional dos resíduos, a floresta deve manter suas características bem semelhantes à floresta original, apesar de menos rica no estoque adulto, em termos econômicos.

PALAVRAS-CHAVE

Composição florística, exploração de impacto reduzido, parcelas permanentes, intensidade de exploração florestal, Amazônia.

\section{Changes on floristic composition after logging in a terra firme forest in the region of Paragominas, $P A$}

\begin{abstract}
The objective of this research was to evaluate changes on floristic composition considering two intensities of harvest in 108 ha of primary forest in the Rio Capim forest management unit, which belongs to Cikel Brasil Verde Madeiras Ltda., in the municipality of Paragominas, PA. Data were collected in two occasions, before logging (2003) and after logging (2004) in $3650 \mathrm{~m} \times 50$ m permanent sample plots, randomly distributed in the area, being 12 in unlogged forest, 12 in logged forest with cutting of boles and 12 in logged forest with cutting of boles plus harvest of coarse woody debris. All trees with $D B H \geq 10 \mathrm{~cm}$ were registered. Before logging occurred 4469 trees in the 36 plots (9 ha sample), belonging to 46 families, 138 genera and 228 species. After logging were recorded 4330 trees but two species disappeared (Licaria sp. e Nectandra sp.). Floristic composition, in the two logging intensities, had significant changes due to the reduced impact logging applied in the area. However, there was no changes significant between the two logged areas, indicating that the harvest of the coarse woody debris after logging had not caused significant damage to the remaining forest. Floristic composition changed slightly after logging but changes were not significant among the three communities, suggesting that even with the intensity of logging applied plus the harvest of coarse woody debris the forest could maintain its characteristics very similar to the original forest, in spite of the stock of trees being economically poorer.
\end{abstract}

\section{KEYWORDS}

Floristic composition, reduced impact logging, permanent sample plot, intensity of logging, Amazonia.

\footnotetext{
1 Engenheira florestal, M.Sc., Projeto Peteco (Embrapa/CNPq). e-mail: lucianafrancez@yahoo.com.br. Trav. Lomas Valentinas, 2717, CEP: 66095-770. Belém-PA (Secretaria Executiva de Ciência, Tecnologia e Meio Ambiente - SECTAM/ Divisão Agroflorestal).

2 Engenheiro florestal, D. Phil., EMBRAPA Amazônia Oriental. e-mail: olegario.carvalho@gmail.com

${ }^{3}$ Engenheiro florestal, Dr. Universidade Federal Rural da Amazônia. e-mail: fernando.jardim@ufra.edu.br
} 


\section{INTRODUÇÃO}

A floresta amazônica distingue-se não só por sua extensão territorial, mas pela alta biodiversidade, pelo elevado potencial econômico e pela rápida destruição de extensas áreas, determinadas por fatores antrópicos, como o extrativismo vegetal e a agricultura de subsistência (Rabelo et al., 2002).

A exploração florestal na Amazônia, antes da década de 1970, tinha sua maior pressão em florestas de várzea, porém, com o passar do tempo, e em conseqüência da falta de tecnologia e da abertura de grandes rodovias, essa atividade passou a ser mais intensa nas áreas de colonização (Pandolfo, 1978). A extração de madeira, praticada em pequena escala, geralmente de forma extremamente seletiva, causava pequeno impacto ecológico (Souza Júnior et al., 1997). Atualmente no Estado do Pará, como no restante da Amazônia, a exploração madeireira tem sido feita de forma predatória, causando impactos severos ao ecossistema florestal (Veríssimo et al., 2002).

Um dos maiores problemas para a definição de práticas de manejo sustentado em floresta tropical úmida está na determinação da intensidade de exploração. Se por um lado a exploração deve ser economicamente viável, por outro, os danos à floresta devem ser mínimos (Carvalho et al.2004).

A pesquisa florestal deve buscar um sistema adequado de manejo florestal a fim de somar valores aos produtos e subprodutos florestais. Os estudos devem ser baseados na ecologia das comunidades, assim como no aproveitamento de subprodutos como os resíduos lenhosos da exploração florestal.

Devido a crescente pressão do mercado consumidor de madeira e da opinião pública no que se refere à sustentabilidade dos recursos florestais, há um número cada vez maior de iniciativas de manejo florestal de baixo impacto que visa reduzir os danos causados à natureza pela interferência humana.

As informaçôes obtidas através dos inventários florestais, sobre a estrutura e composição das florestas, são as maiores ferramentas de que dispōem os engenheiros florestais, para avaliação do potencial e definição de estratégias para o manejo (Sandel \& Carvalho, 2000).

O conhecimento da dinâmica da composição florística é um elemento importante para a elaboração do plano de manejo florestal (Costa et al., 2002). A dinâmica da composição florística é analisada através da distribuição dos indivíduos em espécies, gêneros e famílias botânicas, que ocorrem na área e sua variação no decorrer do período (Sandel \& Carvalho, 2000).

Este estudo teve como objetivo comparar as mudanças ocorridas devido a exploração florestal de impacto reduzido, com e sem a retirada de resíduos lenhosos, na composição florística de uma floresta de terra firme na região de Paragominas, Estado do Pará (PA).

\section{MATERIAL E MÉTODOS}

A área de estudo localiza-se na Fazenda Rio Capim, pertencente a Cikel Brasil Verde Madeiras Ltda., no município de Paragominas. O município está situado no nordeste Paraense, mesorregião Sudeste Paraense e microrregião de Paragominas, entre as coordenadas de $2^{\circ} 25^{\prime}$ e $4^{\circ} 09^{\prime} \mathrm{S}$ e $46^{\circ} 25^{\prime}$ e $48^{\circ} 54^{\prime} \mathrm{W}$ $\mathrm{Gr}$, tendo sua sede localizada no entroncamento do $\mathrm{Km} 0$ da PA 256 com o Km 15 da PA 125 às margens da rodovia BR -010 (IBGE, 1991; Bastos et al. 1993; Leal, 2000).

Segundo a classificação de Köeppen, o clima predominante na região é do tipo "Aw", isto é, tropical chuvoso com estação seca bem definida, caracterizado por temperatura média anual de $27,2^{\circ} \mathrm{C}$, com umidade do ar relativa de $81 \%$ e precipitação pluviométrica com média de $1766 \mathrm{~mm} / \mathrm{ano}$, com ocorrência de menos disponibilidade hídrica no período de julho a outubro (Watrin \& Rocha, 1992).

A área apresenta uma topografia que vai de plana a suavemente ondulada, sendo identificada como pertencente à Região Geomorfológica Planalto Setentrional Pará-Maranhão (Brasil, 1973). O município é drenado por duas bacias, a do rio Capim e a do rio Gurupi, servindo este último de divisa com o Estado do Maranhão (Watrin \& Rocha, 1992).

As principais classes de solos, identificados por Silva (1997) na região, são: Latossolo Amarelo, Podzólico Amarelo e Glei Pouco Húmico, além dos Argissolos encontrados por Brasil (1973 e 1974).

A vegetação da área estudada foi classificada e caracterizada de acordo com Veloso et al. (1991), nos seguintes ambientes fitoecológicos: floresta ombrófila densa, também conhecida como floresta equatorial úmida de terra firme; floresta ombrófila aberta mista de cipó e palmeira; e floresta ombrófila densa aluvial, conhecida também como floresta equatorial úmida de várzea. A tipologia na área de estudo segundo Veloso et al. (1991) éfloresta ombrófila densa submontana (floresta equatorial úmida de Terrafirme).

A pesquisa foi realizada na Unidade de Trabalho No 02 (UT 02), com 108 hectares, na Unidade de Produção Anual № 07 (UPA 07) do Plano de Manejo Florestal da Fazenda Rio Capim.

Foram estabelecidos dois tratamentos, tendo como base a intensidade de colheita de madeira, e uma testemunha $\left(\mathrm{T}_{0}\right)$ para monitorar a floresta não-explorada. O Tratamento $1\left(\mathrm{~T}_{1}\right)$ consiste na exploração de impacto reduzido com a retirada apenas dos fustes das árvores comerciais e o Tratamento $2\left(\mathrm{~T}_{2}\right)$ consiste na exploração de impacto reduzido com a retirada dos fustes das árvores comerciais mais a retirada dos resíduos lenhosos (galhos, restos de troncos, árvores tombadas durante a derruba, partes de troncos não aproveitáveis durante o traçamento) para serem aproveitados na produção de lenha e carvão.

A exploração foi realizada igualmente em toda a área, com exceção das amostras-testemunhas, seguindo as diretrizes 
estabelecidas no plano de manejo da empresa. Após a exploração, foram retirados os resíduos das parcelas sorteadas para o Tratamento $2\left(\mathrm{~T}_{2}\right)$. Foram exploradas, em média, 4,33 árvores/ ha de 17 espécies comerciais. $\mathrm{O}$ volume de fustes retirado foi de $44,67 \mathrm{~m}^{3} \cdot \mathrm{ha}^{-1}$.

Para o monitoramento da vegetação nos 108 ha, foram estabelecidas, aleatoriamente, 36 parcelas quadradas de $0,25 \mathrm{ha:}$ doze para estudar a floresta não-explorada; doze para a área explorada com retirada apenas do fuste comercial das árvores; e doze para a área explorada com retirada do fuste comercial e retirada do resíduo lenhoso. Adotou-se a metodologia de inventário florestal contínuo sugerida por Silva \& Lopes (1984), revisada e atualizada por Silva et al. (2005), tanto para instalação como medição das árvores nas parcelas. Cada parcela de 0,25 ha foi dividida em 25 subparcelas de $10 \times 10 \mathrm{~m}$, onde foram medidos, com fita diamétrica, todos os indivíduos arbóreos com $\mathrm{DAP} \geq 10 \mathrm{~cm}$.

Nas árvores com fuste normal e sem sapopemas, o diâmetro foi medido a 1,30m do solo. Nas árvores com sapopemas, nós, calosidades, podridão, danos ou qualquer deformação a $1,30 \mathrm{~m}$, a medição foi feita em outra posição no fuste, sem influência dessas irregularidades.

A identificação dos indivíduos foi realizada, na floresta, por parabotânicos experientes da Embrapa Amazônia Oriental e da empresa CIKEL Brasil Verde Madeiras Ltda. Foi coletado material botânico das espécies menos comuns e das espécies que suscitaram dúvidas, para a identificação através da comparação, no Herbário IAN da Embrapa Amazônia Oriental.

Foi elaborada uma lista de todas as espécies arbóreas ocorrentes na área de estudo, contendo nome comum, nome científico e família, assim como o número de gêneros e espécies. Foi determinado, também, o quociente de mistura de Jentsch (número médio de plantas para cada espécie), para cada tratamento e testemunha, assim como o índice de Shannon que determina a diversidade de espécies de uma dada comunidade. Para o processamento dos dados utilizaram-se os programas: MFT (Monitoramento de Florestas Tropicais), desenvolvido pela Embrapa Amazônia Oriental, que tem por objetivo analisar os parâmetros referentes à florística e a estrutura da floresta; e o BioEstat 4.0 (Ayres et al., 2005) para a avaliação estatística do estudo.

\section{RESULTADOS E DISCUSSÃO}

\section{COMPOSIÇÃO FLORÍSTICA ANTES DA EXPLORAÇÃO}

Em 2003 foram registrados 4469 indivíduos com DAP $\geq$ $10 \mathrm{~cm}$, nas 36 parcelas amostradas (nove hectares), distribuídos em 46 famílias botânicas, 138 gêneros e 228 espécies (Tabela 1). Das 228 espécies, 28 foram identificadas somente até o nível de gênero, devido à impossibilidade da coleta de material botânico na época de floração ou frutificação, dificultando a comparação dessas espécies em herbário.

Antes da exploração florestal (2003) não houve diferença significativa entre as médias dos tratamentos quanto ao número de espécies, ao nível de $95 \%$ de probabilidade, de acordo com os resultados do teste $t$ aplicado para amostras independentes entre os pares de tratamentos $\left(\mathrm{T}_{0} \mathrm{e} \mathrm{T}_{1} ; \mathrm{T}_{0} \mathrm{eT}_{2} ; \mathrm{T}_{1} \mathrm{eT}_{2}\right)$.

O número de espécies arbóreas (228) encontrado neste estudo foi o mesmo registrado por Wright et al. (1997), ao inventariarem árvores e lianas ( $\mathrm{DAP} \geq 10 \mathrm{~cm}$ ) em uma área de um hectare em Papua Nova Guiné, embora o número de famílias naquela área (58) tenha sido superior ao do presente estudo (48). Oliveira \& Amaral (2004), em área de um hectare, encontraram 239 espécies incluindo árvores, palmeiras e lianas com DAP $\geq 10 \mathrm{~cm}$, distribuídas em 120 gêneros e 50 famílias em floresta de terra firme a noroeste de Manaus. Vale ressaltar que o presente estudo não leva em consideração indivíduos pertencentes ao grupo de lianas.

Oliveira (1994), realizando um estudo sobre a composição florística e potencial madeireiro, dos indivíduos com DAP $\geq 30$ cm, em uma área de 195 ha de floresta no Estado do Acre, identificou 125 espécies pertencentes a 93 gêneros e 45 famílias. Maciel et al. (2000), analisando uma área de 51 ha, descreveram os parâmetros fitossociológicos de uma floresta tropical de terra firme em Caxiuanã, considerando os indivíduos com DAP $\geq 25$ cm, registraram 189 espécies distribuídas em 135 gêneros e 46 famílias. Ballée \& Campbell (1990), amostrando indivíduos com DAP $\geq 10 \mathrm{~cm}$ em duas áreas (Araweté e Asurini) de florestas localizadas próximo ao Rio Xingu - PA, encontraram 36 famílias, 89 gêneros e 142 espécies em 1 ha localizado em Araweté e 38 famílias, 86 gêneros e 137 espécies em Asurini.

Esses estudos realizados no Acre, Caxiuanã e Xingu apresentaram um número de espécies inferior quando comparado com a floresta do presente estudo. Contudo vale ressaltar que para os estudos realizados por Oliveira (1994) e Maciel et al. (2000), não foram considerados os indivíduos com DAP < 30 $\mathrm{cm}$ e com DAP $<25 \mathrm{~cm}$, respectivamente.

Tabela 1 - Número de indivíduos (N) com DAP $\geq 10 \mathrm{~cm}$, famílias (F), gêneros $(G)$ e espécies (E) ocorrentes em uma amostra de 9 ha de floresta de terra firme na Fazenda Rio Capim, Paragominas, PA, antes e após a exploração florestal.

\begin{tabular}{ccccccccccc}
\hline & \multicolumn{3}{c}{ Antes da exploração } & \multicolumn{4}{c}{ Após a exploração } \\
\cline { 2 - 10 } Tratamentos & N & F & G & E & N & F & G & E \\
\hline $\begin{array}{c}\text { Floresta não-explorada } \\
\text { (Testemunha - T0) }\end{array}$ & 1474 & 44 & 121 & 191 & 1485 & 43 & 120 & 190 \\
$\begin{array}{c}\text { Floresta explorada com colheita } \\
\text { apenas do fuste (T1) }\end{array}$ & 1448 & 44 & 114 & 174 & 1374 & 44 & 111 & 168 \\
$\begin{array}{c}\text { Floresta explorada com colheita } \\
\text { apenas do fuste + retirada de } \\
\text { resíduos (T2) }\end{array}$ & 1547 & 43 & 111 & 168 & 1471 & 43 & 111 & 163 \\
$\begin{array}{c}\text { Floresta não-explorada (T0) + } \\
\text { Floresta explorada (T1 + T2) }\end{array}$ & 4469 & 46 & 138 & 228 & 4330 & 46 & 138 & 226 \\
\hline
\end{tabular}


Entretanto, Faber-Langendoen \& Gentry (1991) avaliaram a composição florística, em uma floresta na Colômbia, situada na região do Chocó (Bajo Calima), para árvores com $\mathrm{DAP} \geq 10 \mathrm{~cm}$ em uma parcela de 1 ha e registraram 252 espécies e 45 famílias. Lima Filho et al. (2001), ao inventariar três hectares de floresta ombrófila densa de terra firme no Estado do Amazonas, registraram 2241 indivíduos, com DAP $\geq 10 \mathrm{~cm}$, pertencentes às classes de árvores, palmeiras e cipós, distribuídos em 60 famílias, 225 gêneros e 577 espécies. Esses resultados mostram que na Colômbia e no Amazonas ocorreram 24 e 349 espécies a mais, respectivamente, do que na Fazenda Rio Capim. Portanto a floresta situada na região do Chocó na Colômbia apresenta uma diversidade florística superior aos valores encontrados por Lima Filho et al. (2001) e aos do presente estudo.

As famílias Leguminosae, Lecythidaceae, Sapotaceae e Violaceae destacaram-se em abundância, constituindo juntas $66,27 \%$ dos indivíduos da área não-explorada, 62,90\% da área explorada com retirada apenas do fuste e $63,84 \%$ da explorada com colheita do fuste mais a retirada dos resíduos. Essas famílias destacaram-se, também, em estudos de composição florística realizados por Sandel \& Carvalho (2000) em uma área de cinco hectares de mata alta na Floresta Nacional do Tapajós, onde Leguminosae, Violaceae, Lecythidaceae, Moraceae, Sapotaceae e Burseraceae foram as mais abundantes, constituindo 57,95\% dos indivíduos inventariados.

Oliveira \& Amaral (2004), ao estudarem a florística e a fitossociologia em uma floresta de vertente no Estado do Amazonas, observaram que as famílias mais importantes, em ordem decrescente de número de indivíduos com DAP $\geq 10 \mathrm{~cm}$ foram: Lecythidaceae (118), Burseraceae (87), Sapotaceae (78), Chrysobalanaceae (72), Euphorbiaceae (62) e Fabaceae (43), juntas essas seis famílias responderam por quase $60 \%$ dos espécimes inventariados.

Amaral et al. (2000), em um estudo realizado em floresta densa de terra firme no Estado do Amazonas, verificaram que as famílias com maiores números de indivíduos foram, nesta ordem: Lecythidaceae, Burseraceae, Chrysobalanaceae, Caesalpiniaceae e Sapotaceae.

Lima Filho et al. (2001), em um inventário em floresta de terra firme no Estado do Amazonas, observaram que as famílias Lecythidaceae, Sapotaceae, Chrysobalanaceae, Myristicaceae e Moraceae foram as mais representativas, em número de indivíduos. Ainda no Estado do Amazonas, Matos \& Amaral (1999) constataram que as famílias com maiores abundâncias, em uma floresta de terra firme, foram Lecythidaceae, Lauraceae, Burseraceae, Chrysobalanaceae, Sapotaceae, Annonaceae e Arecaceae totalizando 59\% dos indivíduos registrados. Porém, é importante ressaltar que nesses trabalhos além dos indivíduos arbóreos, foram considerados, também, os indivíduos pertencentes às classes de palmeiras e cipós, o que não foi abordado neste estudo.
Percebe-se que, nesses estudos realizados no Estado do Amazonas, as famílias Lecythidaceae, Sapotaceae, Chrysobalanaceae estavam entre as mais abundantes. No presente estudo, as famílias Lecythidaceae e Sapotaceae também se destacaram quanto ao número de indivíduos, entretanto Chrysobalanaceae não aparece entre as dez mais abundantes.

As famílias Leguminosae ( $\mathrm{T}_{0}=34, \mathrm{~T}_{1}=29, \mathrm{~T}_{2}=30$ espécies $)$ Sapotaceae $\left(\mathrm{T}_{0}=25, \mathrm{~T}_{1}=20, \mathrm{~T}_{2}=21\right.$ espécies $)$, Moraceae $\left(\mathrm{T}_{0}=\right.$ $12, T_{1}=9, T_{2}=10$ espécies $)$, Lecythidaceae $\left(T_{0}=10, T_{1}=9, T_{2}=\right.$ 10 espécies), Lauraceae ( $\mathrm{T}_{0}=8, \mathrm{~T}_{1}=12, \mathrm{~T}_{2}=10$ espécies) e Euphorbiaceae $\left(\mathrm{T}_{0}=6, \mathrm{~T}_{1}=6, \mathrm{~T}_{2}=5\right.$ espécies $)$ apresentaram os maiores números de espécies (Tabela 2), de acordo com Whitmore (1990), as famílias Leguminosae, Annonaceae, Euphorbiaceae, Lauraceae, Moraceae, Myristicaceae, Rubiaceae e Sapotaceae são as mais representativas nas regiōes tropicais. Afirma, também, que a família Lecythidaceae é bem representada na América, especialmente em se tratando de Brasil, com 11 gêneros e aproximadamente 120 espécies.

De acordo com Gentry (1986), embora cada local possua um conjunto de espécies diferentes, a composição dessas florestas em nível de família é similar. Muniz et al., (1994a) acrescentou que cada família deve ter uma regra específica nas comunidades neotropicais, com um grupo diferente de espécies para diferentes substratos na Amazônia.

$\mathrm{Na}$ área de 51 ha, analisada por Maciel et al. (2000), na Floresta Nacional de Caxiuanã, as famílias com maiores números de espécies foram: Leguminosae com 45 espécies (considerando as três subfamílias), seguida de Moraceae com 13 espécies e Chrysobalanaceae e Euphorbiaceae com 9 espécies, cada uma. No presente estudo, as famílias Leguminosae, Moraceae e Euphorbiaceae reuniram, também, os maiores números de espécies. Chrysobalanaceae ( $\mathrm{T}_{0}=3, \mathrm{~T}_{1}=2, \mathrm{~T}_{2}=3$ espécies $)$, no entanto, não aparece entre as famílias com maior riqueza florística na área.

Tabela 2 - Número de gêneros (GEN) e de espécies (ESP) das famílias mais importantes, considerando indivíduos com DAP $\geq 10 \mathrm{~cm}$, em uma área de 108 ha (amostra de 3 ha por tratamento) de floresta de terra firme, Paragominas, PA, antes da exploração.

\begin{tabular}{ccccccc}
\hline \multirow{2}{*}{ Famílias } & \multicolumn{2}{c}{ T0 } & \multicolumn{3}{c}{ T1 } & \multicolumn{2}{c}{ T2 } \\
\cline { 2 - 7 } & GEN & ESP & GEN & ESP & GEN & ESP \\
\hline Euphorbiaceae & 6 & 6 & 6 & 6 & 5 & 5 \\
Lauraceae & 4 & 8 & 5 & 12 & 4 & 10 \\
Lecythidaceae & 4 & 10 & 3 & 9 & 3 & 10 \\
Leguminosae & 21 & 34 & 20 & 29 & 21 & 30 \\
Moraceae & 8 & 12 & 7 & 9 & 6 & 10 \\
Sapotaceae & 5 & 25 & 4 & 20 & 5 & 21 \\
\hline
\end{tabular}

$T_{0}$ - Floresta não-explorada; $T_{1}$ - Colheita apenas dos fustes; $T_{2}$ - Colheita dos fustes + retirada de resíduos lenhosos. 
A família Violaceae, apesar de contribuir em média com 8,01\% da abundância, levando-se em consideração a área testemunha e as áreas com diferentes tratamentos, apresentou dois gêneros e três espécies na área de floresta não-explorada e na floresta explorada com colheita dos fustes e retirada de resíduos, e dois gêneros e duas espécies nas parcelas onde foram apenas colhidos os fustes. Barros et al. (2000b), analisando a fitossociologia de uma floresta situada em Curuá-Una, PA, observaram que a família Violaceae tinha 46,7 árvores por hectare, porém com apenas duas espécies, portanto uma a menos do que no presente estudo. As famílias Moraceae $\left(T_{0}=8\right.$ gêneros e 12 espécies; $T_{1}=7$ gêneros e 9 espécies $\mathrm{e} \mathrm{T}_{2}=6$ gêneros e 10 espécies), e Euphorbiaceae $\left(\mathrm{T}_{0} \mathrm{e} \mathrm{T}_{1}=6\right.$ gêneros e 6 espécies $\mathrm{e} \mathrm{T}_{2}=5$ gêneros e 5 espécies), que possuem um elevado número de espécies, contribuem, em média, com apenas 2,24 e 2,14\%, respectivamente, do total de indivíduos da comunidade, demonstrando que nem sempre a família com maior riqueza de espécies é aquela que possui maior densidade, concordando com Amaral et al. (2000).

As famílias menos abundantes (um indivíduo por família) foram: Araliaceae, Connaraceae, Malpighiaceae, Opiliaceae e Vochysiaceae no $\mathrm{T}_{0}$; Araliaceae, Ebenaceae, Lacistemaceae e Malpighiaceae no $\mathrm{T}_{1}$; e Araliaceae, Bignoniaceae, Caryocaraceae, Ochnaceae, Opiliaceae e Vochysiaceae no $\mathrm{T}_{2}$.

Algumas famílias, no entanto, nem sequer aparecem em determinados tratamentos como é o caso da Combretaceae que ocorreu apenas nas parcelas-testemunhas e Lacistemaceae que só ocorre nas parcelas destinadas à exploração florestal sem a retirada de resíduos. Não foi registrada a ocorrência das famílias Bignoniaceae nas parcelas-testemunhas $\left(\mathrm{T}_{0}\right)$, Vochysiaceae nas parcelas exploradas com retirada apenas do fuste $\left(\mathrm{T}_{1}\right)$ e Connaraceae nas parcelas exploradas com colheita do fuste e dos resíduos lenhosos $\left(\mathrm{T}_{2}\right)$, porém a família que não ocorre em um determinado tratamento ocorre em outro, por exemplo, a família Vochysiaceae não ocorre nas parcelas do $\mathrm{T}_{1}$, entretanto há a ocorrência desta nos demais tratamentos $\left(\mathrm{T}_{0} \mathrm{e} \mathrm{T}_{2}\right)$. As demais famílias são comuns aos três tratamentos.

As espécies com maiores números de indivíduos, nos três tratamentos foram: Rinorea flavescens, Lecythis idatimon, Poecilanthe effusa, Eschweilera grandiflora e Eschweilera pedicellata, ocorrendo em todas as 36 parcelas inventariadas. A espécie Inga sp. apresentou um grande número de indivíduos, porém não ocorreu em quatro das 36 parcelas amostradas.

De acordo com a Tabela 3, observa-se que há uma intensa mistura de espécies na floresta em estudo, sendo semelhante aos citados por Finol (1975), para florestas tropicais, que é de nove plantas por espécie, e por Barros et al. (2000a) que ao estudarem a diversidade de espécies em uma floresta em Curuá-una, diagnosticaram um quociente de 1:12,8 para os indivíduos com $\mathrm{DAP} \geq 45 \mathrm{~cm}$ e 1:12,4 para os indivíduos com DAP $<45 \mathrm{~cm}$.
Sandel \& Carvalho (2000) encontraram um quociente de mistura médio, em uma amostra de 5 ha na Floresta Nacional do Tapajós, de quatro plantas por espécie (1:4). Jardim \& Hosokawa (1986/87), ao realizarem estudos em uma floresta equatorial no Estado do Amazonas, observaram que ao aumentar a amplitude das classes de tamanho a heterogeneidade da floresta também aumentava. O quociente médio de mistura encontrado por estes autores foi de 1:3 considerando indivíduos com $5 \leq \mathrm{DAP}<20$ $\mathrm{cm}$ e 1:2 considerando indivíduos com DAP $\geq 20 \mathrm{~cm}$. Portanto, foram valores superiores ao encontrado no pressente estudo.

Aárea destinada à colheita dos fustes comerciais mais a retirada dos resíduos lenhosos teve menor heterogeneidade devido ao menor número de espécies e maior número de indivíduos quando comparada a $\mathrm{T}_{0} \mathrm{e} \mathrm{T}_{1}$. Porém, esses valores são superiores aos encontrados por Rabelo et al. (2002), para os indivíduos com $\mathrm{DAP} \geq 5 \mathrm{~cm}$ em duas áreas de florestas estuarinas do Estado do Amapá, que foram de 1:33 em Mazagão e 1:25 em Lontra da Pedreira.

\section{COMPOSIÇÃO FLORISTICA APÓS A EXPLORAÇÃO}

Em 2004, sete meses após a exploração, foram feitos 4531 registros de indivíduos com $\mathrm{DAP} \geq 10 \mathrm{~cm}$, com 4330 vivos, nas 36 parcelas amostradas (nove hectares). Houve o desaparecimento de 2 espécies (Licaria sp. e Nectandra sp.), porém o número de famílias e de gêneros permaneceu o mesmo que em 2003.

Rodrigues et al. (1997), ao realizarem levantamento fitossociológico dos indivíduos com $\mathrm{DAP} \geq 10 \mathrm{~cm}$, em uma área de quatro hectares nos municípios de Acará (amostra de um hectare) eTailândia (amostra de três hectares), onde vêm ocorrendo profundas alterações ambientais devido ao rápido processo de consolidação da rodovia PA 150, registraram 135 espécies distribuídas em 99 gêneros e 39 famílias botânicas. Portanto, no levantamento realizado nos municípios de Acará e Tailândia ocorreram 91 espécies a menos do que no presente estudo.

A redução do número de espécies (duas) foi bem menor do que o relatado por Costa et al. (2002), que ao analisarem a composição florística após a colheita de madeira na Floresta Nacional do Tapajós, no período de 1981 a 1997, observaram que dez espécies desapareceram, considerando indivíduos com

Tabela 3 - Quociente de Mistura de Jentsch, para os indivíduos com DAP $\geq 10$ $\mathrm{cm}$, em uma área de 108 ha (amostra de 3 ha por tratamento), de floresta de terra firme, antes e após a exploração florestal, em Paragominas, PA.

\begin{tabular}{ccccccc}
\hline \multirow{2}{*}{ Tratamento } & \multicolumn{3}{c}{ Antes da exploração } & \multicolumn{3}{c}{ Após a exploração } \\
\cline { 2 - 7 } & NE & NI & J & NE & NI & J \\
\hline T0 & 191 & 1474 & $1: 8$ & 190 & 1485 & $1: 8$ \\
T1 & 174 & 1448 & $1: 9$ & 168 & 1374 & $1: 9$ \\
T2 & 168 & 1547 & $1: 10$ & 163 & 1471 & $1: 10$ \\
\hline
\end{tabular}

J-Quociente de Jentsch; NE - Número de espécies; $\mathrm{NI}$ - número de indivíduos; $\mathrm{T}_{0}$ Floresta não-explorada; $T_{1}$ - Colheita apenas dos fustes; $T_{2}$ - Colheita dos fustes + retirada de resíduos lenhosos. 
$\mathrm{DAP} \geq 15 \mathrm{~cm}$, e que nesse período houve apenas o ingresso de cinco novas espécies. Verificaram, também, que aos dois anos após a exploração foram registradas 164 espécies arbóreas, pertencentes a 50 famílias e 123 gêneros, e que aos dezoito anos após a exploração as 50 famílias permaneceram, porém o número de espécies diminuiu para 160. No presente estudo, não foram verificadas espécies novas, no curto período avaliado, porém é provável que, com o passar do tempo, a composição florística seja dinâmica.

Vidal et al. (1998), ao analisarem o efeito da exploração predatória e da planejada sobre a diversidade de espécies em Paragominas - PA notaram que a exploração planejada provocou a redução de 4,0\% (seis espécies) no número de espécies, logo após a exploração. $\mathrm{Na}$ área com exploração predatória essa redução foi de 7,4\% (nove espécies). Os resultados encontrados no presente estudo foram semelhantes aos de Vidal et al. (1998) na área em que foi realizada a exploração florestal planejada.

Foram observados 62 indivíduos novos, alocados da seguinte forma: 21 na área testemunha; 11 na área explorada com colheita apenas dos fustes - $T_{1}$; e 30 na área explorada com colheita dos fustes mais a retirada dos resíduos $-\mathrm{T}_{2}$ (Figura 1). Porém, nas parcelas onde foi realizada a colheita de madeira essa percepção não foi possível, pois o número de indivíduos mortos foi superior ao número de indivíduos novos.

Considerando os indivíduos nos três tratamentos (Tabela 1), houve mudanças quanto ao número de famílias, gêneros e espécies, em relação à medição anterior (antes da exploração). Entretanto através do teste t para amostras independentes, entre os pares dos tratamentos $\left(\mathrm{T}_{0} \mathrm{e} \mathrm{T}_{1} ; \mathrm{T}_{0} \mathrm{e} \mathrm{T}_{2} ; \mathrm{T}_{1} \mathrm{e} \mathrm{T}_{2}\right)$, não houve diferença significativa entre as médias quanto ao número de espécies, ao nível de $95 \%$ de probabilidade. Porém, para amostras pareadas houve diferença significativa para os tratamentos $1\left(\mathrm{~T}_{1}\right.$ - 2003 e $\left.\mathrm{T}_{1}-2004\right)$ e $2\left(\mathrm{~T}_{2}-2003\right.$ e $\left.\mathrm{T}_{2}-2004\right)$. Essa diferença é explicada pelo desaparecimento de espécies nestes tratamentos.

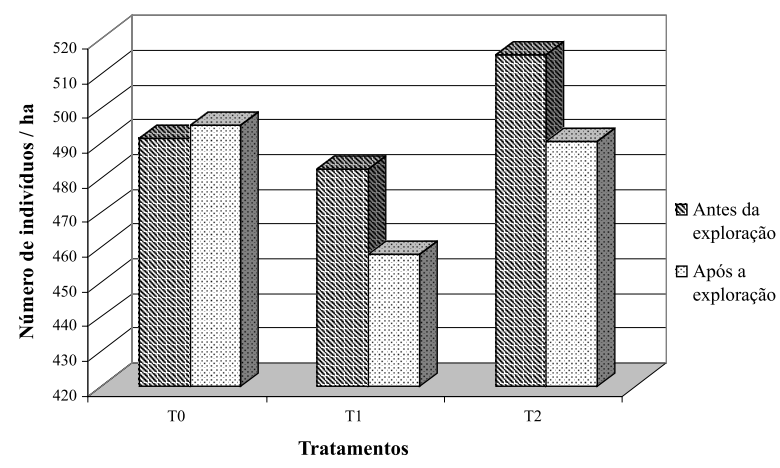

Figura 1 - Número de indivíduos com DAP $\geq 10 \mathrm{~cm}$ em uma amostra de 9 ha, um mês antes e sete meses após a exploração florestal realizada na UT 02, UPA 07 (108 ha), na Fazenda Rio Capim, Paragominas, PA.
Foi detectado o desaparecimento de uma família que possuía apenas uma espécie nas parcelas-testemunhas (Vochysiaceae Qualea paraensis), mas que continua ocorrendo no tratamento 2. Nas parcelas em que foi realizada apenas a colheita dos fustes $\left(\mathrm{T}_{1}\right)$, desapareceram seis espécies (Aspidosperma megalocarpum, Laetia procera, Copaifera multijuga, Stryphnodendron pulcherrimum, Sapium sp. e Licaria sp.). No $\mathrm{T}_{2}$ (colheita dos fustes + resíduos) as espécies Stryphnodendron adstringens, Perebea mollis, Parkia gigantocarpa, Simarouba amara, Pourouma sp. e Nectandra sp. desapareceram após a colheita. Porém, observouse que na área do $\mathrm{T}_{2}$ apareceu uma nova espécie (Sorocea sp.) pertencente à família Moraceae, que já havia ocorrido nas parcelastestemunhas e nas parcelas do $\mathrm{T}_{1}$.

Carvalho (2002), em pesquisa realizada na Floresta Nacional do Tapajós, constatou mudança na composição florística aos sete anos após a exploração com o aparecimento de seis espécies. Avaliando, também, a composição florística da área testemunha (não-explorada) verificou, após seis anos de observações, a diminuição no número de famílias de 48 para 46, o aparecimento de três novas espécies e o desaparecimento de oito espécies. Foi verificado, ainda, que as maiores mudanças ocorreram no tratamento com maior intensidade de exploração. No presente estudo houve a diminuição de uma família em um período de sete meses, na área não-explorada.

Muniz et al. (1994b), ao estudarem a fitossociologia de uma reserva florestal, que vem sofrendo grandes alteraçōes devido aos constantes desmatamentos, em São Luís do Maranhão, verificaram que a família Leguminosae apresentou os maiores parâmetros fitossociológicos da área estudada, resultados diferentes dos encontrados no presente estudo, onde a família Lecythidaceae é a que apresenta os maiores parâmetros fitossociológicos.

Na família Violaceae surgiram dez indivíduos novos após a exploração, representando $16,12 \%$ do total de ingressos do povoamento. Apesar disso, a família se manteve com o mesmo número de gêneros e espécies nos diferentes tratamentos.

As famílias com menores abundâncias em 2004, após a exploração, foram as mesmas de 2003, com apenas uma planta por família.

As espécies com maiores números de indivíduos em 2003, antes da exploração, (Rinorea flavescens, Lecythis idatimon, Poecilanthe effusa, Eschweilera grandiflora, Eschweilera pedicellata, Inga sp.) foram as que mais contribuíram com o ingresso de indivíduos à comunidade, com 46,77\% (29 plantas) do total de indivíduos novos nas três áreas estudadas (Tabela 4). Porém, não é possível visualizar estes indivíduos novos na Tabela 4, pois a mortalidade $(4,5 \%)$ foi superior aos ingressos $(1,4 \%)$ em relação ao total de indivíduos (9 ha) após a exploração.

No presente estudo, verificou-se que, mesmo após a exploração florestal, o quociente de mistura manteve-se alto, semelhante à medição de 2003, quando a floresta ainda não 
Tabela 4 - Número de plantas, com DAP $\geq 10 \mathrm{~cm}$, das seis espécies mais abundantes, antes e após a exploração florestal $\left(\mathrm{T}_{1}\right.$ e $\left.\mathrm{T}_{2}\right)$ em uma amostra de 6 ha de floresta de terra firme, e em duas ocasiões em 3 ha de floresta não-explorada $\left(\mathrm{T}_{0}\right)$, no município de Paragominas, $\mathrm{PA}$.

\begin{tabular}{ccccccc}
\hline \multirow{2}{*}{ Espécies } & \multicolumn{3}{c}{ Antes da exploração } & \multicolumn{3}{c}{ Após a exploração } \\
\cline { 2 - 7 } & T0 & T1 & T2 & T0 & T1 & T2 \\
\hline Rinorea flavescens & 121 & 111 & 109 & 125 & 107 & 103 \\
\hline Lecythis idatimon & 113 & 107 & 140 & 113 & 101 & 125 \\
\hline Poecilanthe effusa & 106 & 102 & 123 & 108 & 98 & 116 \\
\hline Eschweilera grandiflora & 77 & 66 & 83 & 78 & 63 & 81 \\
\hline Eschweilera pedicellata & 64 & 73 & 74 & 61 & 73 & 73 \\
\hline Inga sp. & 58 & 58 & 65 & 58 & 57 & 66 \\
\hline Total & 539 & 517 & 594 & 543 & 499 & 564 \\
\hline
\end{tabular}

havia sido explorada (Tabela 3). Nas parcelas exploradas o número de indivíduos diminuiu, entretanto foi mantida uma proporcionalidade, em relação aos quocientes de mistura, entre uma medição e outra, devido a diminuição do número de espécies. Estes resultados demonstram que o grau de perturbação na área foi relativamente baixo, reafirmando que a exploração de impacto reduzido é menos prejudicial à floresta do que a exploração tradicional quando comparada a outros trabalhos (Vidal et al. 1998; Pantoja et al. 1997; Veríssimo et al. 1992; Uhl et al. 1991).
Pantoja et al. (1997), em um estudo realizado em uma floresta secundária no município de Benevides, PA, encontraram um quociente de 1:23 para a área estudada, demonstrando que esta foi altamente perturbada, diferentemente dos resultados encontrados neste estudo. Afirmaram que o valor baixo para o quociente de mistura demonstra o alto grau de perturbação da floresta.

Os valores de diversidade de espécies para toda a comunidade, assim como para cada tratamento, calculados pelo índice de Shannon e índice de equabilidade, são apresentados na Tabela 5.

O índice de diversidade de Shannon para as 36 parcelas estudadas (área não-explorada + área explorada) em 2003 (antes da exploração) foi de 4,29 com o índice de equabilidade de 0,79. Em 2004 (após a exploração) o índice de diversidade foi de 4,27 com equabilidade de 0,81. Estes valores de diversidade estão dentro dos limites esperados para florestas tropicais, que é de 3,83 a 5,85, de acordo com Knight (1975). Isso não ocorreu em duas microrregiōes do Estado do Pará, onde Ribeiro et al. (1999) encontraram índices de diversidade de Shannon de 3,66 para a microrregião de Carajás e 3,71 para a microrregião de Marabá, o que é explicado devido ao fato do número de espécies ser inferior nessas duas áreas quando comparadas aos valores encontrados no presente estudo.

Com a realização do teste t para amostras pareadas $\left(\mathrm{T}_{0}\right.$ 2003 e $\mathrm{T}_{0}-2004 ; \mathrm{T}_{1}-2003$ e $\mathrm{T}_{1}-2004 ; \mathrm{T}_{2}-2003$ e $\mathrm{T}_{2}$ 2004) e teste t para amostras independentes (antes e após a exploração) entre os pares de tratamentos $\left(\mathrm{T}_{0} \mathrm{e} \mathrm{T}_{1} ; \mathrm{T}_{0} \mathrm{eT} ; \mathrm{T}_{1} \mathrm{e}\right.$

Tabela 5 - Índice de diversidade de Shannon $\left(\mathrm{H}^{\prime}\right)$ e índice de equabilidade $(\mathrm{E})$ para 36 parcelas (amostra de 3 ha em cada tratamento) mensuradas para os indivíduos com DAP $\geq 10 \mathrm{~cm}$ antes (2003) e depois (2004) da exploração florestal de impacto reduzido, na Fazenda Rio Capim, Paragominas, PA.

\begin{tabular}{|c|c|c|c|c|c|c|c|c|c|c|c|c|c|c|}
\hline \multirow{3}{*}{ Parcelas } & \multicolumn{4}{|c|}{ T0 } & \multirow{3}{*}{ Parcelas } & \multicolumn{4}{|c|}{$\mathrm{T} 1$} & \multirow{3}{*}{ Parcelas } & \multicolumn{4}{|c|}{$\mathrm{T} 2$} \\
\hline & \multicolumn{2}{|c|}{2003} & \multicolumn{2}{|c|}{2004} & & \multicolumn{2}{|c|}{2003} & \multicolumn{2}{|c|}{2004} & & \multicolumn{2}{|c|}{2003} & \multicolumn{2}{|c|}{2004} \\
\hline & $\mathrm{H}^{\prime}$ & $E$ & $\mathrm{H}^{\prime}$ & $E$ & & $\mathrm{H}^{\prime}$ & $E$ & $\mathrm{H}^{\prime}$ & $E$ & & $\mathrm{H}^{\prime}$ & $E$ & $\mathrm{H}^{\prime}$ & \\
\hline 1 & 3,48 & 0,87 & 3,48 & 0,87 & 10 & 3,74 & 0,90 & 3,71 & 0,90 & 2 & 3,54 & 0,89 & 3,52 & 0,90 \\
\hline 3 & 3,55 & 0,89 & 3,55 & 0,89 & 15 & 3,49 & 0,90 & 3,50 & 0,90 & 6 & 3,77 & 0,94 & 3,69 & 0,93 \\
\hline 4 & 3,66 & 0,93 & 3,67 & 0,93 & 19 & 3,62 & 0,89 & 3,60 & 0,89 & 12 & 3,43 & 0,88 & 3,49 & 0,90 \\
\hline 5 & 3,66 & 0,90 & 3,62 & 0,89 & 20 & 3,70 & 0,91 & 3,55 & 0,91 & 17 & 3,72 & 0,92 & 3,62 & 0,92 \\
\hline 7 & 3,72 & 0,91 & 3,70 & 0,91 & 26 & 3,28 & 0,88 & 3,03 & 0,88 & 18 & 3,32 & 0,87 & 3,29 & 0,87 \\
\hline 8 & 3,41 & 0,89 & 3,42 & 0,89 & 30 & 3,52 & 0,90 & 3,55 & 0,90 & 22 & 3,35 & 0,84 & 3,36 & 0,84 \\
\hline 9 & 3,51 & 0,89 & 3,53 & 0,89 & 31 & 3,69 & 0,91 & 3,59 & 0,91 & 23 & 3,60 & 0,89 & 3,54 & 0,89 \\
\hline 11 & 3,79 & 0,92 & 3,77 & 0,92 & 32 & 3,52 & 0,91 & 3,45 & 0,91 & 24 & 3,70 & 0,91 & 3,65 & 0,91 \\
\hline 13 & 3,67 & 0,91 & 3,66 & 0,91 & 33 & 3,75 & 0,91 & 3,73 & 0,91 & 25 & 3,84 & 0,93 & 3,80 & 0,92 \\
\hline 14 & 3,65 & 0,90 & 3,63 & 0,90 & 34 & 3,41 & 0,89 & 3,41 & 0,90 & 27 & 3,59 & 0,91 & 3,60 & 0,91 \\
\hline 16 & 3,52 & 0,89 & 3,51 & 0,89 & 35 & 3,81 & 0,93 & 3,82 & 0,93 & 28 & 3,28 & 0,86 & 3,34 & 0,89 \\
\hline 21 & 3,72 & 0,93 & 3,74 & 0,93 & 36 & 3,53 & 0,92 & 3,53 & 0,92 & 29 & 3,79 & 0,93 & 3,76 & 0,92 \\
\hline TOTAL & 4,29 & 0,82 & 4,27 & 0,81 & & 4,25 & 0,82 & 4,21 & 0,82 & & 4,14 & 0,81 & 4,12 & 0,81 \\
\hline
\end{tabular}


$\mathrm{T}_{2}$ ), verificou-se que não houve diferença significativa tanto antes como após a exploração florestal a 95\% de probabilidade.

O índice de diversidade da floresta não-explorada $\left(\mathrm{T}_{0}\right)$ foi de 4,29 e 4,27 para a primeira e segunda medição, respectivamente, considerando todas as parcelas juntas (amostra de $3 \mathrm{ha}$ ). Os valores da equabilidade foram relativamente altos, em torno de 0,82 (Tabela 5).

Maciel et al. (2000) determinaram um índice de diversidade de Shannon de 3,71 e equabilidade de 0,71 , para árvores com $\mathrm{DAP} \geq 25 \mathrm{~cm}$, em uma área da Floresta Nacional de Caxiunã. Barros et al. (2000a) encontraram um índice de diversidade, em uma floresta não-explorada, na região de Curuá-una, de 3,86 para os indivíduos com DAP $\geq 45 \mathrm{~cm}$ e 3,206 para os indivíduos com $10 \mathrm{~cm} \leq \mathrm{DAP}<45 \mathrm{~cm}$. Tais valores estão abaixo do encontrado para o total das parcelas-testemunhas, no presente trabalho. Vale ressaltar, porém, que os limites de inclusão dos DAP são diferentes.

Nas parcelas do $T_{1}$ (colheita dos fustes), analisadas conjuntamente, os índices de diversidade foram 4,25 e 4,21 antes e após a exploração, respectivamente. $\mathrm{O}$ índice de diversidade de Shannon no $T_{2}$ (colheita de fustes + colheita de resíduos) foi de 4,14 e 4,12 antes e após a exploração, respectivamente. Portanto, menor do que no $\mathrm{T}_{1}$ e no $\mathrm{T}_{0}$. Esses valores são considerados altos quando comparados ao índice de 2,91 reportados por Pantoja et al. (1997) para um trecho de floresta secundária em Benevides demonstrando que a exploração feita de forma planejada acarreta menos prejuízos à floresta remanescente.

Vidal et al. (1998), ao estudarem os efeitos da exploração predatória e da planejada sobre a diversidade de espécies em Paragominas - PA perceberam diferença altamente significativa dentro dos tratamentos, onde foram realizadas a exploração planejada e a exploração predatória, o que não ocorreu na áreatestemunha. Porém, três anos após a exploração, essa diferença ocorreu apenas nas parcelas em que foi realizada a exploração florestal predatória.

Os índices de diversidade de Shannon determinados no presente estudo são semelhantes ou, em alguns casos, até maiores do que os reportados em outros trabalhos realizados na região amazônica, demonstrando que a área em estudo está dentro dos padrōes das florestas primárias de terra firme da Amazônia, mesmo que haja diferenças na composição florística das diferentes amostras, o que é bastante plausível, considerando a alta heterogeneidade das florestas localizadas na região amazônica.

A equabilidade de todas as parcelas foi superior a 0,8 , isso significa que não há a dominância de uma espécie ou de um pequeno grupo de espécies na comunidade, indicando que a comunidade florística da área em estudo está prestes a atingir a diversidade máxima, onde cada indivíduo pertence a uma espécie diferente, caracterizando alta heterogeneidade florística
(Magurran, 1988). Esse fato foi detectado por alguns autores, em estudos sobre a estrutura da floresta e a florística na Amazônia, demonstrando que geralmente nas florestas de terra firme ocorre alta diversidade, ou seja, poucos indivíduos em cada espécie (Oliveira \& Mori 1999; Lima Filho, 2001).

\section{CONCLUSÕES}

A composição florística da floresta não-explorada sofreu alteraçōes no período estudado, embora sem significância estatística, demonstrando que a floresta natural está sempre em dinamismo, ainda que com mínimas mudanças.

A composição florística, tanto na área onde foram colhidos apenas os fustes comerciais como na área onde, além da colheita dos fustes, foram também colhidos os resíduos lenhosos $\left(\mathrm{T}_{2}\right)$, sofreu alteraçôes significantes em consequiência da exploração florestal de impacto reduzido de 26 indivíduos de 17 espécies comercias a que foram submetidas, ao se comparar as diferentes mediçōes (antes e após a exploração florestal) para um mesmo tratamento. Entretanto, não houve alterações significantes entre as duas áreas, demonstrando que, em termos ecológicos, a retirada dos resíduos lenhosos após a colheita dos fustes não vai implicar em danos significativos à floresta residual.

Após a exploração florestal, a composição florística e a diversidade, mesmo com pequenas alterações, não mostraram significância entre os três tratamentos (floresta não-explorada, floresta onde houve colheita de fustes comerciais, e floresta onde houve colheita dos fustes comerciais e dos resíduos lenhosos), sugerindo que com a intensidade de exploração baixa e mesmo com a retirada adicional dos resíduos, a floresta deve continuar com suas características bem semelhantes à floresta original, apesar de um pouco menos rica em estoque adulto, em termos econômicos.

\section{BIBLIOGRAFIA CITADA}

Amaral, I.L.; Matos, F.D.A.; Lima, J. 2000. Composição florística e parâmetros estruturais de um hectare de floresta densa de terra firme no Rio Uatumã, Amazônia, Brasil. Acta Amazonica, 30(3): 377-392.

Ayres, M.; Ayres Júnior, M.; Ayres, D. L.; Santos, A. de A. S. dos. 2005. BioEstat - Aplicaçôes estatísticas nas áreas das ciências BioMédicas. Belém: Imprensa Oficial do Estado do Pará, 324pp.

Ballée, W.; Campbell, G.D. 1990. Evidence for the successional status of liana forest (Xingu River Basin, Amazonian Brrazil). Biotropica, 22(1): 36-47.

Barros, A.V.; Barros, P.L.C.; Silva, L.C.B. 2000a. Estudo da diversidade de espécies de uma floresta situada em Curuá-UnaPará. Revista de Ciências Agrárias, 33: 49-65.

Barros, A.V.; Barros, P.L.C.; Silva, L.C.B. 2000b. Análise fitossociológica de uma floresta situada em Curuá-Una - Pará. Revista de Ciências Agrárias, 34: 9-36. 
Bastos, T.X.; Rocha, A.M.A.; Pacheco, N.A.; Sampaio, S.M.N. 1993. Efeito da remoção da floresta ombrófila sobre regime pluviométrico no município de Paragominas - PA. Boletim de Geografia Teorética, 23 (45/46): 85-92.

Brasil. Departamento Nacional de Produção Mineral. Levantamento de recursos minerais. 1973. Folha SA.23 - São Luís e parte da folha SA.24 - Fortaleza. Geologia, geomorfologia, solos, vegetação e uso potencial da terra.Vol.3. Ministério de Minas e Energia, Rio de Janeiro.

Brasil. Departamento Nacional de Produção Mineral. Levantamento de recursos minerais. 1974. Folha SA.22 - Belém. Geologia, geomorfologia, solos, vegetação e uso potencial da terra. Vol.5. Ministério de Minas e Energia, Rio de Janeiro.

Carvalho, J.O.P. 2002. Changes in the floristic composition of a terra firme rain forest in Brazilian Amazonia over an eight-year period in response to logging. Acta Amazonica, 32(2): 277-291.

Carvalho, J. O. P. de; Silva, J. N. M.; Lopes, J. do C. A. 2004. Growth rate of a terra firme rain forest in Brazilian Amazonia over an eight-year period in response to logging. Acta Amazonica, 34 (2): 209-217.

Costa, D.H.M.; Carvalho, J.O.P.; Silva, J.N.M. 2002. Dinâmica da composição florística após a colheita de madeira em uma área de terra firme na Floresta Nacional do Tapajós (PA). Revista de Ciências Agrárias, 38: 67-90.

Faber-Langendoen, D.; Gentry, A.H. 1991. The structure and diversity of rain forest at Bajo Calima, Chocó, region, western Colombia. Biotropica, 23(1): 2-11.

Finol, U.H. 1975. La silvicultura en la Orinoquia Venezolana. Revista Forestal Venezolana, 18(25): 37-114.

Gentry, A.H. 1986. An overview of neotropical phytogeographic patterns with an emphasis on Amazonia. In: Anais do I Simpósio do Trópico Úmido. EMBRAPA/CPATU, Belém. p.19-35.

Ibge. Instituto Brasileiro de Geografia e Estatística. 1991. Sinopse preliminar do censo demográfico 1991. IBGE, Rio de Janeiro. 74pp.

Jardim, F.C.S.; Hosokawa, R.T. 1986/87. Estrutura da floresta equatorial úmida da estação experimental de silvilcultura tropical do INPA. Acta Amazonica, 16/17: 411-508.

Knight, D. H. 1975. A phytosociological analysis of species-rich tropical forest on Barro Colorado Island, Panamá. Ecological Monographs, 45: 259-284.

Leal, G. L. R. 2000. Paragominas: A realidade do pioneirismo. Alves. Belém. 498pp.

Lima Filho, D.A.; Matos, F.D.A.; Amaral, I.L.; Revilla, J.; Coelho, L.S.; Ramos, J.F.; Santos, J.L. 2001. Inventário florístico de floresta ombrófila densa de terra firme, na região do Rio UrucuAmazonas, Brasil. Acta Amazonica, 31: 565-579.

Maciel, M.N.M.; Queiroz, W.T.; Oliveira, F.A. 2000. Parâmetros fitossociológicos de uma floresta tropical de terra firme na floresta nacional de Caxiuanã (PA). Revista de Ciências Agrárias, 34: 85106.

Magurran, A. E. 1988. Ecological Diversity and its Measurements. New Fatter Lane: London. 179pp.
Matos, F.D.A.; Amaral, I.L. 1999. Análise ecológica de um hectare em floresta ombrófila densa de terra firme, Estrada de Várzea, Amazonas, Brasil. Acta Amazonica, 29(3): 365-379.

Muniz, F.H.; Cesar, O.; Monteiro, R. 1994a. Aspectos florísticos quantitativos e comparativos da vegetação arbórea da Reserva Florestal do Sacavém, São Luís, Maranhão (Brasil). Acta Amazonica, 24(3/4): 189-218.

Muniz, F.H.; Cesar, O.; Monteiro, R. 1994b. Fitossociologia da vegetação arbórea da Reserva Florestal do Sacavém, São Luís, Maranhão (Brasil). Acta Amazonica, 24(3/4): 219-236.

Oliveira, A.N.; Amaral, I.L. 2004. Florística de uma floresta de vertente na Amazônia Central, Amazonas, Brasil. Acta Amazonica, 34(1): 21-34.

Oliveira, A. A.; Mori, S. A. 1999. A Central Amazonian terra firme forest. I. High tree species richness on poor soils. Biodiversity and Conservation, 8: 1219-1244.

Oliveira, M.V.N.. 1994. Composição florística e potenciais madeireiro e extrativista em uma área de floresta no Estado do Acre. EMBRAPA - CPAF (EMBRAPA - CPAF Boletim de Pesquisa, 9), Rio Branco. 42pp.

Pandolfo, C. 1978. A floresta amazônica brasileira: enfoque econômico e ecológico. SUDAM, Belém. 77pp.

Pantoja, F.B.C.; Oliveira, V.C.; Costa, L.G.S.; Vasconcelos, P.C.S. 1997. Estrutura de um trecho de floresta secundária de terra firme, no Município de Benevides, PA. FCAP/ Serviço de documentação e informação (FCAP, Informe Técnico, 24), Belém, PA. 18pp.

Rabelo, F.G.; Zarin, D.J.; Oliveira, F.A.; Jardim, F.C.S. 2002. Diversidade, composição florística e distribuição diamétrica do povoamento com $\mathrm{DAP} \geq 5 \mathrm{~cm}$ em região de estuário no Amapá. Revista de Ciências Agrárias, 37: 91-112.

Ribeiro, R. J.; Higuchi N.; Santos, J. dos; Azevedo, C. P. 1999. Estudo fitossociológico nas regiōes de Carajás e Marabá - Pará, Brasil. Acta Amazonica, 29(2): 207-222.

Rodrigues, I.A.; Pires, J.M.; Watrin, O.S.; Cordeiro, M.R. 1997. Levantamento fitossociológico em áreas sob influência da rodovia PA - 150 nos municípios de Acará e Tailândia, PA. Embrapa Amazônia Oriental (Boletim de pesquisa, 179), Belém. 43pp.

Sandel, M.P.; Carvalho, J.O.P. 2000. Composição florística e estrutura de uma área de cinco hectares de mata alta sem babaçu na Floresta Nacional do Tapajós. Embrapa Amazônia Oriental. Documentos. 63. Belém. 19p.

Silva, J.N.M.; Lopes, J.C.A. 1984. Inventário florestal contínuo em florestas tropicais: a metodologia da EMBRAPA-CPATU na Amazônia brasileira. EMBRAPA-CPATU (EMBRAPA-CPATU. Documentos, 33), Belém.36pp.

Silva, J.N.M.; Lopes, J.C.A.; Oliveira, L.C.; Silva, S.M.A.; Carvalho, J.O.P.; Costa, D.H.M.; Melo, M.S.; Tavares, M.J.M. 2005. Diretrizes para Instalação e Medição de Parcelas Permanentes em Florestas Naturais da Amazônia Brasileira. Embrapa / ITTO, Belém. 68pp. 
Silva, R.C. 1997. Contribuição do levantamento de solo e caracterização dos sistemas naturais e ambientais na regiāo de Paragominas - Estado do Pará. Dissertação de Mestrado, Faculdade de Ciências Agrárias do Pará, Belém, PA. 107pp.

Souza Júnior, C.; Veríssimo, A.; Stone, S.; Uhl, C. 1997. Zoneamento da Atividade Madeireira na Amazônia: Um Estudo de Caso Para o Estado do Pará. n.8. IMAZON (Série Amazônia), Belém. 28pp.

Uhl, C.; Veríssimo, A.; Mattos, M. M.; Brandino, Z.; Vieira, J. C. G. 1991. Social economic and ecological consequences of selective logging in an Amazon frontier: The case of Thailand. Forest Ecology and Management, 46: 243-273.

Vidal, E.; Viana, V.; Batista, J. L. F. 1998. Efeitos da exploração madeireira predatória e planejada sobre a diversidade de espécies na Amazônia Oriental. Revista Árvore, 22(4): 503-520.

Veloso, H.P.; Rangel Filho, A.L.R.; Lima, J.C.A. 1991. Classificação da vegetação brasileira adaptada a um sistema universal. IBGE, Departamento de recursos naturais e estudos ambientais, Rio de Janeiro. 124pp.
Veríssimo, A; Barreto, P.; Mattos, M.; Tarifa, R.; Uhl, C. 1992. Logging impacts and prospects for sustainable forest management in old Amazonia frontier: the case of Paragominas. Forest Ecology and Management, 55: 169-199.

Veríssimo, A.; Lima, E.; Lentini, M. 2002. Pólos madeireiros do Estado do Pará. IMAZON, Belém. 72pp.

Vidal, E.; Viana, V.; Batista, J.L.F. 1998. Efeitos da exploração madeireira predatória e planejada sobre a diversidade de espécies na Amazônia Oriental. Revista Árvore, 22(4): 503-520.

Watrin, O.S.; Rocha, A.M.A. 1992. Levantamento de vegetação natural e uso da terra no Município de Paragominas (PA) utilizando imagens TM/Landsat. EMBRAPA-CPATU (EMBRAPA-CPATU. Boletim de Pesquisa, 124), Belém. 40pp.

Whitmore, T.C. 1990. An introduction to tropical rain forest. Clarendon Press, Oxford. 226pp.

Wright, D.D.; Jessen, J.H.; Burke, P.; Garza, H.G.S. 1997. Tree and liana enumeration and diversity on an one-hectare plot in Papua New Guinea. Biotropica, 29(3): 250-260.

Recebido em 06/10/2006

Aceito em 04/05/2007 